\title{
ESTUDIOS DE CASOS Y CONTROLES
}

\author{
CASE-CONTROL STUDIES \\ Alonso Soto ${ }^{1,2, a, b}$; Aleksandar Cvetkovic-Vega ${ }^{1, a}$
}

\begin{abstract}
RESUMEN
Los estudios de casos y controles son estudios epidemiológicos observacionales analíticos utilizados generalmente para evaluar factores asociados a condiciones de presentación infrecuente. Su jerarquía dentro de la pirámide de evidencia se ubica en un lugar intermedio, generalmente con un nivel de evidencia considerado mayor que los estudios transversales analíticos y menor que los estudios de cohorte. La medida de asociación utilizada para estos estudios es el Odds Ratio (o razón de momios). Algunas variantes de este diseño como los estudios de casos y controles incidentes y los anidados (dentro de una cohorte) permiten disminuir el riesgo de sesgo de selección. Se revisan los conceptos básicos relacionados a este tipo de estudios.
\end{abstract}

Palabras clave: Casos y Control; Epidemiología; Causalidad; Estudio observacional (fuente: DeCS BIREME).

\section{ABSTRACT}

Case-control studies are analytical observational epidemiological studies generally devoted to evaluate factors associated with infrequent diseases. Its hierarchy within the pyramid of evidence lies in an intermediate place between cross sectional and cohort studies. The measure of association used for these studies is the Odds Ratio. Some variants of this design, such as incident and nested case studies and nested case-control studies (within a cohort) can reduce the risk of selection bias. The basic concepts related to this type of studies are reviewed.

Key words: Cases-Control; Epidemiology; Causality; Observational study (source: MeSH NLM).

\section{CONCEPTOS BÁSICOS}

Los estudios de Casos y Controles son estudios epidemiológicos de tipo observacional, dado que no se realiza una intervención sino se "observa" la ocurrencia de eventos, y analíticos puesto que permiten formular una hipótesis en relación a evaluar la asociación entre dos o más variables, teniendo además un grupo contraste denominado en este caso "Control"(1-3).

Su jerarquía dentro de la pirámide de evidencia se ubica en un lugar intermedio, generalmente con un nivel de evidencia considerado mayor que los estudios transversales y menor que los estudios de cohorte. Son útiles para evaluar la relación entre posibles exposiciones y efectos, partiendo de la presencia de un evento de interés y evaluando de forma retrospectiva la temporalidad de la asociación con una o más exposiciones.

En los estudios de casos y controles se parte de la presencia de un evento (EFECTO, denominado también outcome o desenlace) identificando personas o pacientes con la presencia de éste y comparándolos con un grupo de características semejantes, pero sin la presencia del mismo. Las personas con el evento de interés se denominan CASOS, mientras que aquellas personas escogidas para servir como comparadores se denominan CONTROLES. Es importante resaltar 2 aspectos en los controles puesto que es su selección adecuada es clave para este diseño: los controles deben de

${ }^{1}$ Instituto de Investigación en Ciencias Biomédicas, INICIB, Universidad Ricardo Palma, Lima-Perú.

${ }^{2}$ Departamento de Medicina. Hospital Nacional Hipólito Unanue, Lima-Perú.

a Médico internista. PhD in Health Sciences.

${ }^{b}$ Médico cirujano.

Citar como: Soto A; Cvetkovic-Vega A. Estudios de casos y controles. Rev. Fac. Med. Hum. Enero 2020; 20(1):138-143. DOI 10.25176/RFMH.v20i1.2555 
partir de la misma población que dio origen a los casos, y además deben de ser similares a éstos, salvo por la exposición ${ }^{(1)}$.

Una vez identificados los casos y controles, se debe evaluar la presencia de la EXPOSICION en cada uno de los grupos. Es decir, se identifica de forma retrospectiva la temporalidad en la presencia o no de la exposición.
La temporalidad es un punto muy importante para poder diferenciar entre los 3 diseños de estudios observacionales analíticos. Mientras en los estudios de cohortes se va de la exposición al efecto, y en los estudios trasversales éstos se miden en paralelo; en los estudios de casos y controles se parte del efecto hacia la exposición (figura 1).

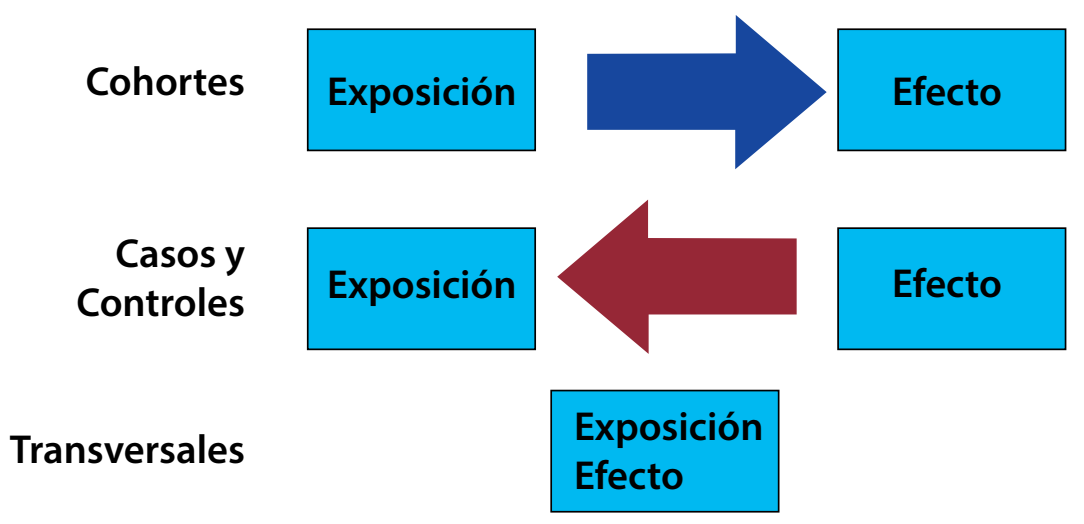

Figura 1. Dirección de la temporalidad en la asociación en estudios de casos y controles, transversales y cohortes.

\section{EL ODDS RATIO COMO MEDIDA DE ASOCIACIÓN EN ESTUDIOS DE CASOS Y CONTROLES}

El Odds es una razón entre dos eventos mutuamente excluyentes. Este vocablo inglés no tiene una traducción específica al español, pero históricamente proviene de las apuestas, en donde la razón es ganar o perder. En investigación científica, se trata de una razón entre la ocurrencia o no de un evento. Por ejemplo, si tenemos 100 personas y 20 de ellas fallecen y los otros 80 sobreviven, el Odds de morir será de 20 a 80 , o $20 / 80=0.25$. Este Odds de morir equivale a una probabilidad de morir de $(20 / 20+80)=0.2$. El Odds entonces es un estimador que tiene correlación con la probabilidad de un evento. En particular tiene cifras cercanas a la probabilidad cuando los Odds (y probabilidades) son bajos, y para efectos de evaluar la asociación entre dos variables podemos comparar los Odds de exposición entre personas con y sin la enfermedad. Esta razón de Odds se denomina Odds Ratio o Razón de momios y es la es la medida de asociación del diseño de tipo caso-control ${ }^{(1,4,5)}$.Esto se presenta gráficamente en la figura 2 .

\begin{tabular}{lcc}
\hline & Casos & Controles \\
Expuestos & A & B \\
No Expuestos & C & D \\
\hline
\end{tabular}

Donde

$A=$ Número de Casos con exposición

$B=$ Número de Controles con exposición

$\mathrm{C}=$ Número de Casos sin exposición

$\mathrm{D}=$ Número de Controles sin Exposición

$A / C=$ Odds de Exposición en Casos

$B / D=O d d s$ de Exposición en Controles

Odds Ratio $=\frac{\text { Odds de exposición en casos }}{\text { Odds de exposición en controles }}$

Figura 2. Razón de Odds de exposición en casos y en controles 
En base a los datos de la figura 2 podemos presentar el Odds Ratio como:

Odds Ratio=Odds de Exposición en Casos/Odds de Exposición en controles

$$
\text { Odds Ratio }=\frac{\mathrm{A} / \mathrm{C}}{\mathrm{B} / \mathrm{D}}
$$

Reordenando dicha razón:

$$
\text { Odds Ratio }=\frac{\mathrm{A}^{*} \mathrm{D}}{\mathrm{B} * \mathrm{C}}
$$

Esta equivalencia es denominada "producto cruzado".

La interpretación general de un Odds ratio es la siguiente

$\mathrm{OR}=1$ Odds de exposiciones igual en casos y controles: No asociación.

OR $<1$ factor asociado a menor Odds del evento (factor protector).

OR $>1$ factor asociado a mayor Odds del evento (factor de riesgo).

Debe sin embargo recordarse que no solo debe evaluarse el valor numérico del Odds Ratio presentado. Es importante evaluar dicha razón en el contexto de su intervalo de confianza y la significancia estadística dada por el valor de $p$.

\section{CONSIDERACIONES PARA EL DISEÑO}

\section{Eficiencia estadística}

Es posible incrementar la eficiencia estadística aumentando el número de controles. Sin embargo, debe considerarse que más allá de 4 controles por caso el incremento en la eficiencia estadística es escaso por lo que no se recomienda sobrepasar dicho número.

\section{Cálculo del tamaño muestral}

Sabemos que para que una muestra sea representativa de la población, y en ese sentido permita hacer inferencias hacia ella, debe de tener tanto un cálculo de tamaño muestral adecuado como un muestreo aleatorizado. En esta sección por fines académicos, presentamos la fórmula para el cálculo de tamaño muestral en este diseño cuando no se parea casos y controles. En la actualidad, los programas estadísticos como STATA y plataformas como Open Epir permiten que los investigadores ahorren tiempo en desarrollar paso a paso los cálculos matemáticos, introduciendo los datos requeridos por el software ${ }^{(6)}$. Sin embargo, esto no debe en ningún motivo hacerse de forma aislada o desligada del conocimiento teórico del diseño y sus aspectos estadísticos.

La fórmula estadística para estudios de tipo CasoControl no pareado es la siguiente ${ }^{(4,7)}$ :

$$
n_{c}=\frac{\left(p_{1} \cdot q_{1}+p_{2} \cdot q_{2}\right) \cdot\left(Z_{a}+Z_{\beta}\right)^{2}}{\left(p_{1}-p_{2}\right)^{2}}
$$

Figura 3. Fórmula estadística para el cálculo de tamaño de muestra en diseños caso-control no pareados.

Donde:

$\mathrm{n}_{\mathrm{c}}=$ Número de casos y número de controles sin ajuste

$\mathrm{p}_{1}=$ Proporción esperada del factor en casos

$\mathrm{q}_{1}=1-\mathrm{p}_{1}$

$\mathrm{p}_{2}=$ Proporción esperada del factor en controles

$\mathrm{q}_{2}=1-\mathrm{p}_{2}$

Tener en cuenta que si existe una relación desigual entre casos y controles, sea el caso que la razón de controles/casos sea diferente de 1 , al resultado de nc debe hacérsele un ajuste:

$$
n_{a}=\frac{{ }^{n} c \cdot(1+c)}{2 c}
$$

Figura 4. Ecuación de ajuste para razones control/ casos diferentes de 1.

Donde:

na $=$ Número de controles ajustado

$\mathrm{nc}=$ Número de controles sin ajuste

$c=$ cociente de dividir los controles entre los casos

\section{Elección de los Casos:}

Casos prevalentes: Son los casos existentes al momento de realizar el estudio. Ejemplo: pacientes infartados en un hospital durante un momento determinado.

Casos incidentes: se realiza un seguimiento prospectivo buscando identificar la aparición de casos. Ejemplo: seguimiento de pacientes que ingresan con dolor torácico e identificación prospectiva de pacientes con infarto. 
Los casos prevalentes muestran un mayor riesgo de sesgo, en particular si el hecho de permanecer o no hospitalizado depende de factores asociados a la exposición de interés. Los casos de menor riesgo pueden ser dados de alta tempranamente, mientras que los casos más graves podrían fallecer y no ser evaluados.

\section{Elección de los Controles}

La elección de los controles es el aspecto metodológico posiblemente más relevante en estudios de casos y controles. Como ya se mencionó anteriormente, volvemos a reincidir en la importancia de que el grupo control provenga de la misma población y que sea semejante al grupo CASO con la única excepción de la exposición. La elección inadecuada del grupo control es una de las principales fuentes de errores sistemáticos en este tipo de diseños.

\section{Controles Hospitalarios vs comunitarios}

En muchos estudios los casos son pacientes que se hospitalizan por alguna condición clínica específica, y los controles deben ser personas que, si hubieran enfermado, serían incluidos como casos. En tal sentido, resulta controversial en muchos casos utilizar controles hospitalarios dado que pueden partir de poblaciones que no necesariamente sean similares a las de la cual provienen los casos. Por ejemplo, en un Centro de Referencia para Tuberculosis que recibe población de muchas de las regiones a nivel nacional, no podríamos utilizar como controles a pacientes de Servicios de Medicina, en cuyo caso el alcance del Servicio puede ser mucho más local. Esto hace que muchas veces se apele al uso de controles provenientes de la comunidad que difieran únicamente de los casos en la presencia del efecto o evento de interés. Una forma de disminuir el riesgo de sesgo en la elección de controles hospitalarios es la utilización del concepto de casos incidentes.

\section{Controles Pareados vs no pareados}

Una de las formas de lograr que los casos y controles sean lo más homogéneos posible es aparearlos en función de ciertas características. El pareamiento será más efectivo si la(s) variable(s) se encuentran relacionadas al resultado de interés. Por ejemplo, en un estudio sobre el efecto de comorbilidad en la mortalidad en pacientes con neumonía, los casos de neumonía pueden aparearse con controles de la misma edad. Dado que la edad es un predictor importante de mortalidad en neumonía, su efecto es removido de la asociación y las diferencias pueden ser más plausiblemente debidas al factor estudiado.
Sin embargo, existen algunas desventajas de parear. En términos de factibilidad, puede ser más laborioso y en ocasiones logísticamente imposible hacer el emparejamiento siendo esto más importante cuanto mas exigente sea el mismo. Por otro lado, las variables utilizadas para parear los casos y controles no pueden ser posteriormente analizadas como posibles predictores o covariables en los modelos ajustados.

\section{Estudios de Casos y Controles Anidados (en una cohorte)}

Estos estudios son de utilidad cuando se trabaja con eventos infrecuentes y en los que la determinación de la exposición o el efecto involucran altos costos, de modo que sólo es factible el estudio de un número limitado de participantes. En este diseño particular de casos y controles, anidado dentro de una cohorte (habitualmente de varios cientos o miles de pacientes), se selecciona a aquellos pacientes que desarrollan el evento y son comparados con controles (habitualmente seleccionados de manera aleatoria entre aquellos participantes que no desarrollan el evento de interés).

\section{ANÁLISIS ESTADÍSTICO}

Los estudios de Casos y Controles al ser estudios observacionales tienen una probabilidad apreciable de presentar confusión, es decir la distorsión de una relación entre la exposición y el efecto debido a una tercera variable (variable confusora) asociada tanto a la exposición como al efecto). Un aspecto importante del análisis de los estudios de casos y controles involucra ajustar el Odds Ratio a la presencia de potenciales confusores. Esto se realiza habitualmente mediante un análisis de regresión logística múltiple binaria(8). Si los hallazgos del análisis crudo y ajustado son consistentes, ello brinda una mayor confiabilidad a los resultados obtenidos. En la tabla 1 se presenta la comparación de los OR crudo (análisis sin ajuste) y OR ajustado. En la columna de la izquierda se aprecia el análisis de la variable 3 (dependiente) para las variables 1 y variables 2 por separado. En la columna de la derecha se aprecia el análisis de la variable 3 contra la variable 1 ajustada por la variable 2 (teniendo en cuenta que el efecto de la variable 2 pueda influir tanto en la 1 como en la 3). En este caso puede observarse que en el análisis ajustado disminuye la magnitud de la asociación a juzgar por la disminución del valor del OR. Debe tenerse en cuenta que las distintas técnicas de regresión dependen en gran parte del número de observaciones y variables incluidas. Asimismo, es conveniente evaluar la bondad de ajuste de los modelos mediante pruebas como la de Hosmer and Lemeshow ${ }^{(8)}$. 
Tabla 1. Comparación de los Odds Ratio crudo y ajustado.

\begin{tabular}{ccccccc} 
& \multicolumn{3}{c}{ Variable 3 (Dependiente) } & \multicolumn{3}{c}{ Variable 3 (Dependiente) } \\
\hline & OR crudo & IC (95\%) & $\mathrm{p}$ & OR ajustado & IC (95\%) & $\mathrm{P}$ \\
Variable 1 & 2.98 & $1.95-4.58$ & 0.001 & 1.83 & $1.51-2.21$ & 0.001 \\
Variable 2 & 0.46 & $0.25-0.85$ & 0.014 & 0.61 & $0.43-0.87$ & 0.006 \\
\hline
\end{tabular}

\section{VENTAJAS Y DESVENTAJAS}

Todos los diseños tienen limitaciones que conllevan a ventajas y desventajas. No existe un diseño perfecto y el investigador debe tener muy presente ello al elegir el mejor diseño para responder a su pregunta de investigación. Se considera una buena práctica en investigación el indicar las limitaciones metodológicas del diseño, de manera que los demás investigadores tengan conocimiento de éstas cuando se busque replicar el estudio.

Los estudios de casos y controles son estudios en general relativamente sencillos y baratos. Su ejecución es relativamente rápida. Los estudios de casos y controles permiten la evaluación de múltiples exposiciones y el escenario más apropiado es el de condiciones (efectos) infrecuentes o con periodos de latencia prolongados.

La principal desventaja del estudio de caso y controles es el sesgo de selección ${ }^{(1)}$. Otro sesgo frecuente es el sesgo de recuerdo(1) que generalmente se produce cuando los controles recuerdan con mayor probabilidad una posible exposición.

Por otro lado, una limitación importante de los estudios de casos y controles es que no permite la estimación de prevalencias, dado que la proporción de personas con y sin el evento es fija, siendo determinada por el investigador en el cálculo del tamaño muestral al introducir la razón entre expuestos y no expuestos ${ }^{(1)}$. Otra importante limitación es que no es un estudio útil para exposiciones infrecuentes. Finalmente, la medida de asociación utilizada en estos estudios es poco apropiada para el caso de enfermedades frecuentes, ya que tiende a sobrestimar la magnitud del efecto de la asociación en comparación con el riesgo relativo(9). En la figura 5 se aprecia que el OR siempre tiene un valor más extremo que el riesgo relativo, siendo la diferencia más evidente a medida que los valores se alejan más de la unidad.

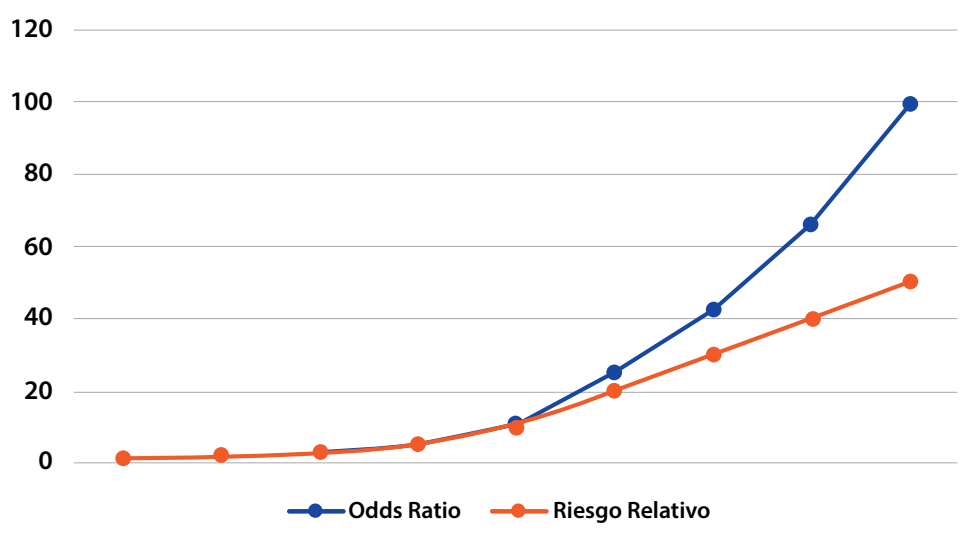

Figura 5. Comparación de Riesgos Relativo y Odds Ratio. 


\section{CONCLUSIÓN}

Los estudios de casos y controles son estudios relativamente fáciles y rápidos en su ejecución. Son una aproximación relativamente sencilla, aunque especialmente sujeta a sesgos (particularmente de selección y de memoria), siendo particularmente útiles en el estudio de condiciones infrecuentes.

La figura muestra el proceso general en el desarrollo de un estudio de caso y controles incluyendo los aspectos resaltantes en la selección de casos, selección de controles, análisis de datos e interpretación de los resultados. Aunque clásicamente se ha ligado este tipo de estudios a un alto riesgo de sesgo, el uso de controles incidentes y los modelos anidados en una cohorte ofrecen una reducción importante del riesgo de sesgo y por lo tanto un mayor nivel de evidencia.
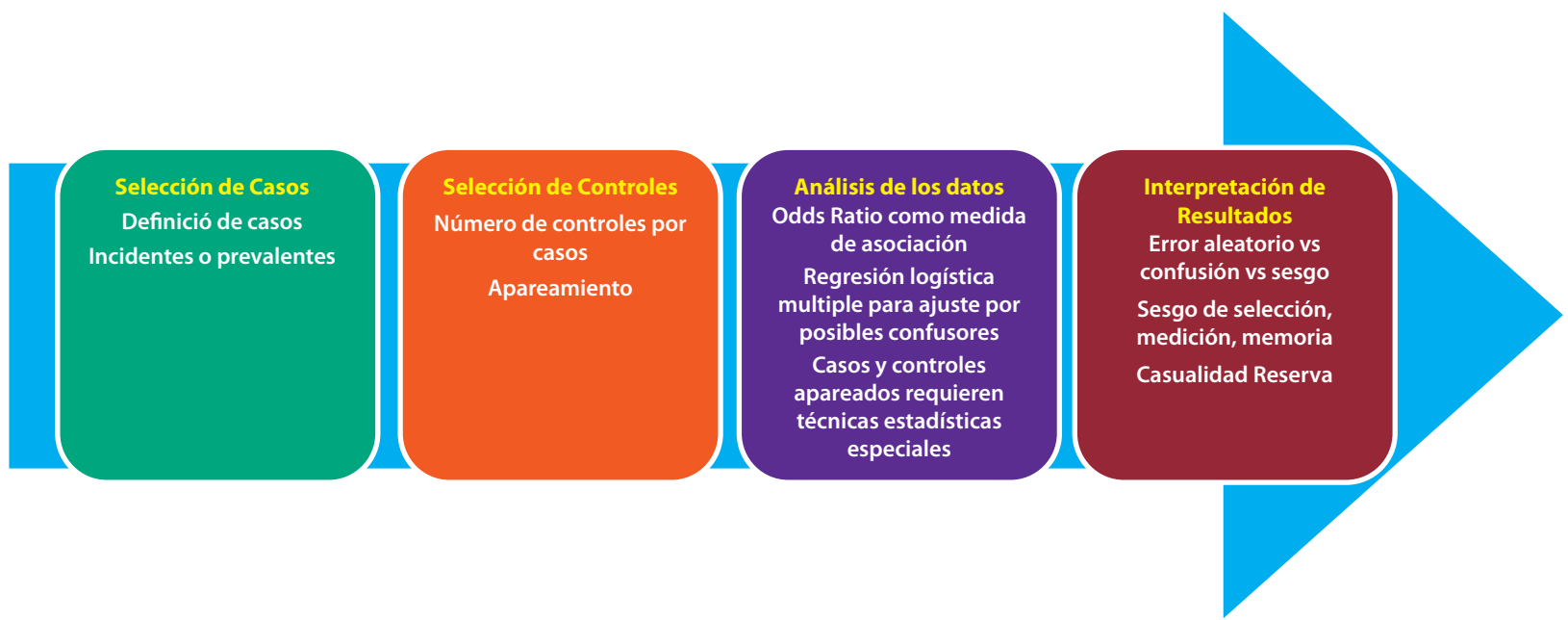

Figura 6. Secuencia del proceso de elaboración de un estudio con diseño de tipo caso-control.

Contribuciones de autoría: AS y AC participaron en la genesis de la idea, la elaboración del manuscrito y la aprobación de su versión final.

Financiamiento: Autofinanciado.

\section{Correspondencia: Alonso Soto.}

Dirección: Instituto de Investigación en Ciencias Biomédicas. Universidad Ricardo Palma. Av Benavides 5440.Santiago de Surco, Lima-Perú.

Teléfono: (01) 7080000

Correo: sotosolari@gmail.com
Conflicto de interés: Los autores declaran no tener ningún conflicto de interés.

Recibido: 15 de noviembre del 2019

Aprobado: 26 de diciembre del 2019

\section{REFERENCIAS BIBLIOGRÁFICAS}

1. Gordis, Leon. Epidemiología. Quinta. Barcelona, España: Elsevier Saunders; 2014. 391 p.

2. Argimon Pallás, Josep M., Jiménez Villa, Josep. Métodos de Investigación Clínica y Epidemiológica. Cuarta. Barcelona, España: Elsevier Saunders; 2013. $402 \mathrm{p}$

3. García Villar C. Introducción a la lectura crítica de artículos: diseño de estudios y sesgos. Radiología [Internet]. enero de 2015 [citado 13 de junio de 2019];57:3-13. Disponible en: https://linkinghub.elsevier.com/retrieve/pii/ S0033833814001441

4. Camacho-Sandoval J. Nota estadística Tamaño de muestra en estudios clínicos. Acta Médica Costarricence. 2008;50(1):2.

5. Cerda J, Vera C, Rada G. Odds ratio: aspectos teóricos y prácticos. Rev Médica Chile [Internet]. octubre de 2013 [citado 13 de junio de 2019];141(10):1329-35 Disponible en: http://www.scielo.cl/scielo.php?script=sci arttext\&pid=S0034 $98872013001000014 \&$ Ing=en\&nrm=iso\&tlng=en
6. Juul S, Frydenberg M. An introduction to Stata for health researchers. 3rd ed. College Station, Tex: Stata Press; 2010. 340 p.

7. Velasco Rodríguez, Víctor, Martínez Ordaz, Verónica, Ruiz Hernándes, José Muestreo y tamaño de muestra: Una guía práctica para personal de salud que realiza la investigación. 1era ed. Coahuila, México: Buenos Aires; 2002.

8. Michael J Campbell. Statistics at Square two- Understanding modern statistical applications in Medicine. 2nd ed. UK: Blackwell; 2006. 133 p.

9. Knol MJ, Le Cessie S, Algra A, Vandenbroucke JP, Groenwold RHH. Overestimation of risk ratios by odds ratios in trials and cohort studies: alternatives to logistic regression. CMAJ Can Med Assoc J [Internet]. 15 de mayo de 2012 [citado 17 de junio de 2019];184(8):895-9. Disponible en: https://www.ncbi.nlm.nih.gov/pmc/articles/PMC3348192/ 